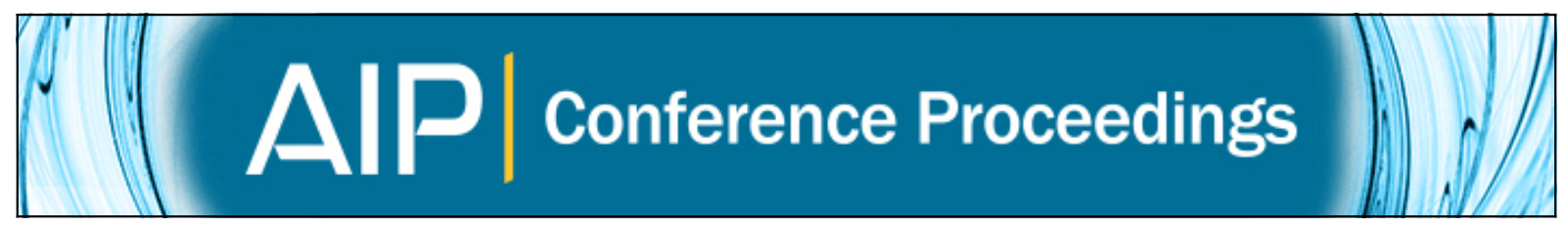

\title{
Excitability of guided waves in composites with PWAS transducers
}

Yanfeng Shen and Victor Giurgiutiu

Citation: AIP Conference Proceedings 1650, 658 (2015); doi: 10.1063/1.4914666

View online: http://dx.doi.org/10.1063/1.4914666

View Table of Contents: http://scitation.aip.org/content/aip/proceeding/aipcp/1650?ver=pdfcov

Published by the AIP Publishing

Articles you may be interested in

Ultrasonic guided wave sensing performance of a magnetostrictive transducer using Galfenol flakes-polymer composite patches

J. Appl. Phys. 117, 17A916 (2015); 10.1063/1.4916811

Efficient excitation of guided acoustic waves in semiconductor nanorods through external metallic acoustic transducer

Appl. Phys. Lett. 105, 243101 (2014); 10.1063/1.4904414

Frequency dependent directivity of guided waves excited by circular transducers in anisotropic composite plates J. Acoust. Soc. Am. 132, EL119 (2012); 10.1121/1.4734392

FE SIMULATION OF GUIDED WAVES IN COMPOSITE MATERIALS GENERATED AND DETECTED BY AIRCOUPLED TRANSDUCERS

AIP Conf. Proc. 1096, 904 (2009); 10.1063/1.3114354

Ultrasonic Guided Wave Imaging of a Composite Plate with Air-Coupled Transducers

AIP Conf. Proc. 894, 1007 (2007); 10.1063/1.2718077 


\title{
Excitability of Guided Waves in Composites with PWAS Transducers
}

\author{
Yanfeng Shen ${ }^{\text {a) }}$ and Victor Giurgiutiu ${ }^{\text {b) }}$ \\ Department of Mechanical Engineering, University of South Carolina, Columbia, South Carolina, United States \\ a)shen5@email.sc.edu \\ b)victorg@sc.edu
}

\begin{abstract}
Piezoelectric Wafer Active Sensors (PWAS) are convenient enablers for generating and receiving ultrasonic guided waves. The wide application of composite structures has put new challenges for the Structural Health Monitoring (SHM) and Nondestructive Evaluation (NDE) community due to the general anisotropic behaviors and complicated guided wave features in composites. The excitability of guided waves in composite structures directly influences the implementation of active sensing systems to achieve the best interrogation of certain sensing directions. This paper presents a hybrid modeling technique for studying the excitably of guided waves in composite structures with PWAS transducers. This hybrid technique comprehensively covers local finite element model (FEM), semi-analytical finite element (SAFE) method, and analytical guided wave solutions. Harmonic analysis of a small-size local FEM with non-reflective boundaries (NRB) was carried out for obtaining guided wave generation features in plate structures. The PWAS transducers were modeled with coupled filed elements. Thus, the FEM can fully capture the geometry and material property effects of PWAS transducers and their influence on the guided wave excitation. SAFE method was used to obtain the complicated guided wave features in composites such as dispersion curves and modeshapes. The SAFE procedure was coded into MATLAB Graphical User Interface (GUI), and the software SAFE-DISPERSION was developed. To study the excitability of each wave mode, we considered all the possible wave modes being generated simultaneously and propagating independently. The analytical wave expressions based on the exact guided wave solution with Hankel functions were used to join the SAFE method and the local FEM. Formulated in frequency domain, the hybrid model is highly efficient, providing an over determined equation system for the calculation of mode participation factors. Case studies were carried out: (1) the Lamb wave excitability in an aluminum plate was investigated and compared with classical pin force models to show the feasibility of the hybrid technique; (2) the guided wave excitability in a woven glass fiber composite (GFRP) plate was studied with circular and square PWAS transducers. The paper finishes with summary, conclusions, and suggestions for future work.
\end{abstract}

\section{INTRODUCTION}

Piezoelectric wafer active sensors (PWAS) are widely explored in structural health monitoring (SHM) and nondestructive evaluation (NDE) procedures for generating and receiving guided waves [1]. Their advantages over conventional ultrasonic transducers are found in their small size and light weight characteristics, making them more suitable for the purpose of permanent installation and real time monitoring. Besides, PWAS transducers work in the in-plane mode and generate long propagating Lamb waves in plates, enabling them to interrogate large areas of structures, while conventional ultrasonic transducers can only perform thickness direction point inspections.

The increasing use of composite components in structures brought new challenges to the SHM and NDE community, because composite materials usually have anisotropic properties and exhibit directional mechanical behaviors. The importance of studying guided wave excitability in composite structures can be seen in two aspects: (1) to optimize directional sensing capability of SHM/NDE system; (2) to reduce sensing signal complexity for ease of signal interpretation. Due to the general anisotropic material properties of composites, guided waves excited by the transducers will be strong in certain directions and week in some others. This effect needs to be fully understood to excite strong interrogating field in the direction of interest. Besides, the nature of guided waves in composites is multimodal and dispersive. This will introduce complicated wave packets in the sensing signals. By choosing the

41st Annual Review of Progress in Quantitative Nondestructive Evaluation
AIP Conf. Proc. 1650, 658-667 (2015); doi: 10.1063/1.4914666

(C) 2015 AIP Publishing LLC 978-0-7354-1292-7/\$30.00 
appropriate PWAS size and excitation frequency, one can diminish the participation of other wave modes, achieving a single wave packet in the interrogating field. This will considerably reduce the sensing signal complexity.

The excitability of guided waves in metallic structures has been explored with analytical approach and experimental verifications $[2,3]$. But this topic becomes more challenging in the case of composites. Analytical approaches have been proposed using the pin-force model and 3-D Green's function method $[4,5]$. But in these models, the distribution of traction field between the PWAS and the plate is hard to be determined and is usually assumed to be pin-forces around PWAS edges. This estimation is accurate when the PWAS thickness is small compared with plate thickness and when the adhesive layer is thin enough to be considered as ideal bonding. In other words, the analytical models cannot capture the local dynamics of the PWAS transducer and the adhesive layer, while these local dynamics may exert considerable influence on the excitability of guided waves. In addition to the analytical methods, numerical modeling techniques provide an alternative way to study this topic. Numerical methods, such as spectral element method (SEM), local interaction simulation approach (LISA), and FEM have been investigated to study the guided wave generation by PWAS transducers $[6,7,8]$. They have shown the capability of capturing the local dynamics of PWAS and adhesive. The first two techniques have not reached their mature stage, while the FEM has been widely examined by experiments for reliable results. However, full scale transient modeling is computational expensive and may become prohibitive for high frequency, short wavelength, long propagating waves in laminate composites. This paper presents a hybrid modeling technique, combining local FEM, SAFE method, and global analytical expression together, to solve the excitability of guided waves in composite structures.

\section{GUIDED WAVES IN COMPOSITE PLATES AND SAFE-DISPERSION}

In this study, the semi-analytical finite element (SAFE) method was adopted to obtain the dispersion curves and modeshapes of composite plates. The SAFE method has been intensively explored as a powerful method to compute the numerical solution of waves in waveguides with arbitrary cross-sections and composite structures [9, 10]. The SAFE method uses finite elements to discretize the cross-section of the waveguide, while the wave propagation direction is expressed with analytically. This semi-analytical formulation finally reaches a stable eigenvalue problem. The eigenvalues are the wavenumbers; the eigenvectors are the corresponding modeshapes. The SAFE procedure was coded using MATLAB Graphical User Interface (GUI), and the predict tool SAFE-DISPERSION was developed to obtain the guided wave solutions for plate structures. Figure 1 shows the material properties input panel and the main interface of SAFE-DISPERSION.
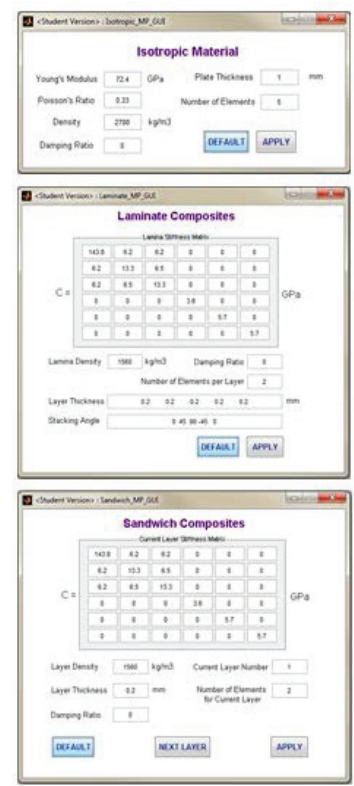

(a)

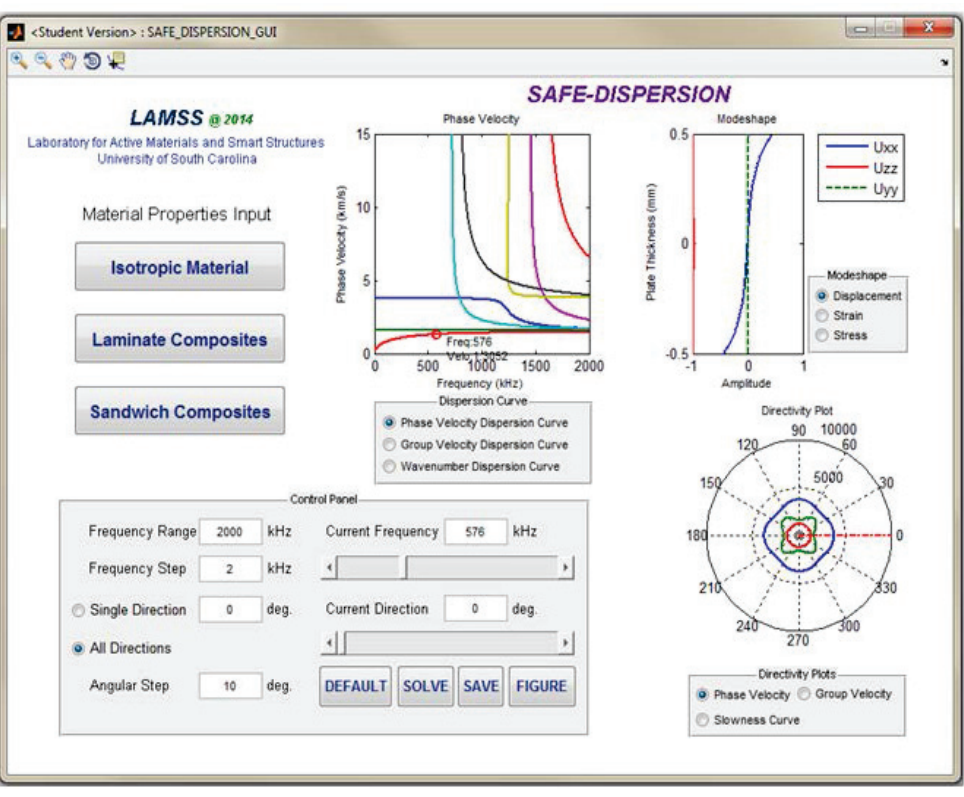

(b)

FIGURE 1. SAFE-DISPERSION GUI: (a) material properties input panel; (b) main interface. 
The composite material in this study is woven glass fiber reinforced polymer (GFRP) which is widely used in naval applications. The material stiffness matrix $C_{G F R P}$ is given in Eq. (1). The density $\rho_{G F R P}$ is $1960 \mathrm{~kg} / \mathrm{m}^{3}$. The laminate composite is 1-mm thick, constructed with 6 woven GFRP plies with the same stacking orientation.

$$
C_{G F R P}=\left[\begin{array}{cccccc}
28.7 & 5.7 & 3 & 0 & 0 & 0 \\
5.7 & 28.7 & 3 & 0 & 0 & 0 \\
3 & 3 & 12.6 & 0 & 0 & 0 \\
0 & 0 & 0 & 4.1 & 0 & 0 \\
0 & 0 & 0 & 0 & 4.9 & 0 \\
0 & 0 & 0 & 0 & 0 & 4.9
\end{array}\right] G P a
$$

Figure 2a shows the SAFE solution of phase velocity dispersion curves in 0 degree direction with respect to fiber orientation. It can be noticed that the solution can capture the symmetric modes, anti-symmetric modes, and shear horizontal modes. The wave velocity varies with propagation direction due to the anisotropic material properties of the woven GFRP plate. The directivity plot of phase velocity at $200 \mathrm{kHz}$ is shown in Fig. $2 \mathrm{~b}$ as an illustrative example. It can be observed that the velocities of symmetric and antisymmetric modes reach maximum along the 0/90 fiber direction and minimum in \pm 45 degree directions, while the shear horizontal mode has highest velocity in \pm 45 degree directions and lowest velocity in $0 / 90$ fiber directions.

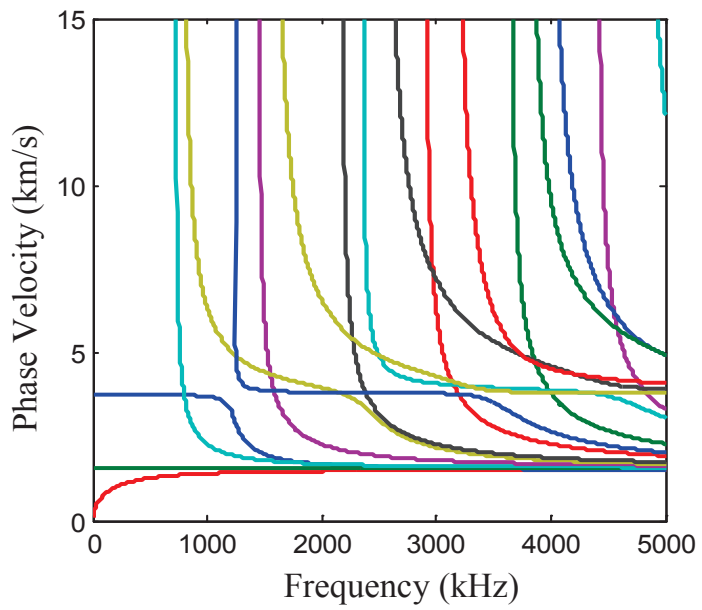

(a)

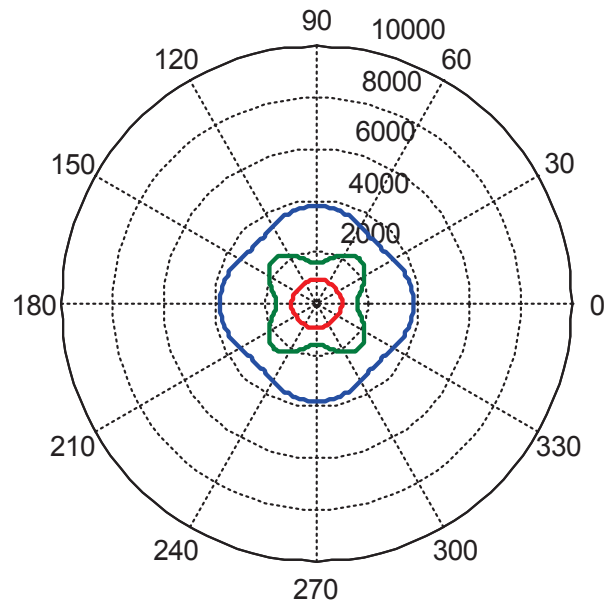

(b)

FIGURE 2. (a) Dispersion curves in 0 degree direction; (2) Phase velocity directivity plot at $200 \mathrm{kHz}$.

\section{SMALL-SIZE FEM WITH NON-REFLECTIVE BOUNDARY}

A small-size FEM with non-reflective boundary (NRB) was used to model the guided wave excitation procedure. Figure 3a shows the schematic of the FEM. The size of the FEM was minimized by adopting NRB and symmetric conditions. Frequency domain harmonic analysis was carried out, so that we can get the solution for all the frequencies of interest with only one run the FEM. The NRB makes it possible to simulate continuous propagating waves rather than standing waves with the small-size FEM. The NRB was constructed using COMBIN14 spring damper elements available in ANSYS. The damping parameter distribution is shown in Fig. 3b. For detailed description of the NRB construction, readers are referred to Ref [11]. The sensing boundary picks up the outward propagating waves arriving at the sensing nodes. PWAS was modeled using the coupled field elements which couple the electrical and mechanical behavior through piezoelectric constitutive equations. Thus, the small-size FEM can capture the complex PWAS dimension and the local dynamics of PWAS and adhesive layer. The model is highly efficient due to its small-size and frequency domain solving scheme. 


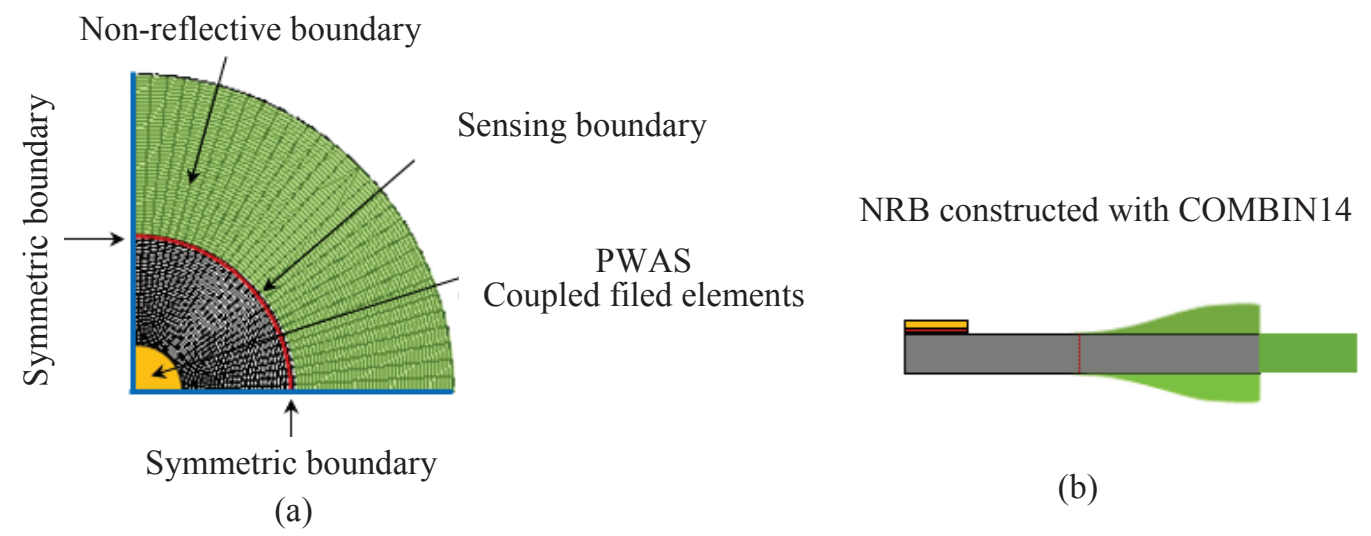

FIGURE 3. (a) Small-size FEM with NRB; (b) NRB constructed with COMBIN14 spring-damper elements.

\section{EXTRACTION OF EXCITABILITY INFORMATION FROM SMALL-SIZE FEM}

The difficulty of extracting excitability information from the small-size FEM is that the wave motion is no longer uncoupled among the wave modes in composites. For instance, the modeshape of SH0 mode shows not only the primary $u_{\theta}$ displacement component, but also the $u_{r}$ component. S0 mode shows the primary $u_{r}$ displacement component, the $u_{\theta}$ component, and also $u_{z}$ component as well.

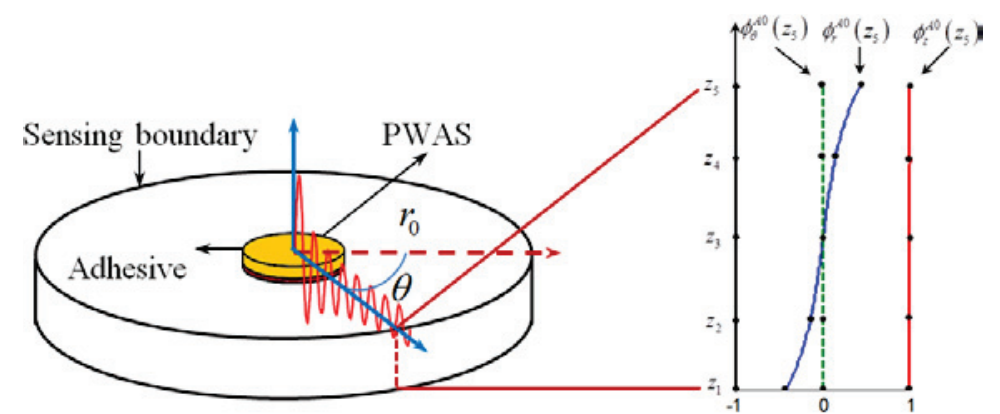

FIGURE 4. Extraction of excitability information from the small-size FEM.

To extract the excitability information, we assumed that the guided wave modes are generated by PWAS and propagate into the structure simultaneously as shown in Fig. 4. The outward propagating 2-D wave field has been shown to follow Hankel function pattern $[1,12]$. The total displacement components picked up at the sensing boundary are the superposition of displacements from all the wave modes. For example, $U_{r}^{F E M}(z)$ represents the $u_{r}$ displacement component from FEM solution at a thickness location $z$ and consists of the superposition of $u_{r}$ components contributed by S0, A0, and SH0 mode. At discretized thickness-wise locations, this relationship can be expressed as shown in Eq. (2) below, i.e.,

$$
\left\{\begin{array}{c}
\sum_{m=1}^{M} a_{m} \phi_{r}^{m}\left(z_{1}\right) H_{1}^{(1)}\left(\xi^{m} r_{0}\right)=U_{r}^{F E M}\left(z_{1}\right) \\
\sum_{m=1}^{M} a_{m} \phi_{r}^{m}\left(z_{2}\right) H_{1}^{(1)}\left(\xi^{m} r_{0}\right)=U_{r}^{F E M}\left(z_{2}\right) \\
\vdots \\
\sum_{m=1}^{M} a_{m} \phi_{r}^{m}\left(z_{n}\right) H_{1}^{(1)}\left(\xi^{m} r_{0}\right)=U_{r}^{F E M}\left(z_{n}\right)
\end{array}\right.
$$


where $a_{m}$ is the mode participation factor of the $m^{\text {th }}$ wave mode; $\phi_{r}^{m}\left(z_{n}\right)$ is the $u_{r}$ component of the $m^{\text {th }}$ wave modeshape at location $z_{n} ; H_{1}^{(1)}$ is Hankel function of the first kind and order one describing an outward propagating 2-D wave field; $\xi^{m}$ is the wavenumber of the $m^{\text {th }}$ wave mode; $r_{0}$ is the distance between the PWAS center and the sensing boundary. Similar relationship can be obtained for $u_{\theta}$ and $u_{z}$ displacement components. These relationships are described using the analytical expressions. The modeshapes and wavenumbers can be calculated using the SAFE method. The $U_{r}^{F E M}(z)$ are calculated from the small-size FEM. Thus, we arrive at a series of linear equations with the mode participation factors as the only unknown quantities. Since the number of equations is greater than the number of unknowns and increases as we further discretize the thickness direction in the small-size FEM, we finally obtain an over-determined equation system, which is solved with least square method using MATLAB. The equation system is expressed in matrix form as

$$
\Phi \cdot H \cdot A=U^{F E M}
$$

where $\Phi$ is the modeshape matrix; $H$ is the 2-D wave propagation matrix; $A$ is the unknown mode participation factor vector to be solved; $U^{F E M}$ is the FEM solution vector. These matrices and vectors are given as follows.

$$
\begin{aligned}
& \Phi=\left[\begin{array}{cccc}
\phi_{r}^{1}\left(z_{1}\right) & \phi_{r}^{2}\left(z_{1}\right) & \cdots & \phi_{r}^{m}\left(z_{1}\right) \\
\vdots & & & \vdots \\
\phi_{r}^{1}\left(z_{n}\right) & \phi_{r}^{2}\left(z_{n}\right) & \cdots & \phi_{r}^{m}\left(z_{n}\right) \\
\phi_{\theta}^{1}\left(z_{1}\right) & \phi_{\theta}^{2}\left(z_{1}\right) & \cdots & \phi_{\theta}^{m}\left(z_{1}\right) \\
\vdots & & & \vdots \\
\phi_{\theta}^{1}\left(z_{n}\right) & \phi_{\theta}^{2}\left(z_{n}\right) & \cdots & \phi_{\theta}^{m}\left(z_{n}\right) \\
\phi_{z}^{1}\left(z_{1}\right) & \phi_{z}^{2}\left(z_{1}\right) & \cdots & \phi_{z}^{m}\left(z_{1}\right) \\
\vdots & \vdots & & \vdots \\
\phi_{z}^{1}\left(z_{n}\right) & \phi_{z}^{2}\left(z_{n}\right) & \cdots & \phi_{z}^{m}\left(z_{n}\right)
\end{array}\right] \\
& H=\left[\begin{array}{cccc}
H_{1}^{(1)}\left(\xi^{1} r_{0}\right) & 0 & \cdots & 0 \\
0 & H_{1}^{(1)}\left(\xi^{2} r_{0}\right) & 0 & \vdots \\
\vdots & 0 & \vdots & 0 \\
0 & \cdots & 0 & H_{1}^{(1)}\left(\xi^{m} r_{0}\right)
\end{array}\right] \\
& A=\left[\begin{array}{llll}
a_{1} & a_{2} & \cdots & a_{m}
\end{array}\right]^{T} \\
& U^{F E M}=\left[\begin{array}{llll}
U_{r}^{F E M}\left(z_{1}\right) \cdots U_{r}^{F E M}\left(z_{n}\right) & U_{\theta}^{F E M}\left(z_{1}\right) \cdots U_{\theta}^{F E M}\left(z_{n}\right) & U_{z}^{F E M}\left(z_{1}\right) \cdots U_{z}^{F E M}\left(z_{n}\right)
\end{array}\right]^{T}
\end{aligned}
$$

The displacement amplitude for the $m^{\text {th }}$ wave mode can be calculated using the calculated mode participation factor and the corresponding modeshape.

$$
u_{r}^{m}=\left|a_{m} \phi_{r}^{m}(z)\right| ; \quad u_{\theta}^{m}=\left|a_{m} \phi_{\theta}^{m}(z)\right| ; \quad u_{z}^{m}=\left|a_{m} \phi_{z}^{m}(z)\right|
$$




\section{CASE STUDIES}

Three groups of cases studies will be shown based on the hybrid method described in the previous sections: (1) excitability of guided waves by a circular PWAS on a 1-mm thick aluminum plate verified with analytical solution; (2) excitability of guided waves by a circular PWAS on a 1-mm thick woven GFRP plate; (3) excitability of guided waves by a rectangular PWAS on a 1-mm thick woven GFRP plate.

\section{Circular PWAS on a 1-mm Thick Aluminum Plate}

In this case study, five situations are considered: (1) pin force excitation; (2) $0.2 \mathrm{~mm}$ thick PWAS with ideal bonding condition; (3) $0.2 \mathrm{~mm}$ thick PWAS with $30 \mu \mathrm{m}$ adhesive layer; (4) $0.2 \mathrm{~mm}$ thick PWAS with $60 \mu \mathrm{m}$ adhesive layer; (5) $0.5 \mathrm{~mm}$ thick PWAS with $30 \mu \mathrm{m}$ adhesive layer. The pin force excitation model is used to compare with the analytical solution given in Eq. (9). For more details of the derivation, readers are referred to Ref [1]. The PWAS and the adhesive layer thickness changes aim at demonstrating the capability of the hybrid model to capture the local dynamics of the transducer and bonding agent. The density, Young's modulus, and Poisson's ratio of the bonding layer were taken as $\rho=1700 \mathrm{~kg} / \mathrm{m}^{3}, E=5 \mathrm{GPa}$, and $v=0.4$ as suggested by Ref [13].

$$
\left.u_{r}(r)\right|_{z=d}=-\pi i \frac{a^{2} \tau_{a}}{2 \mu} \sum_{\xi^{S}} \frac{J_{1}\left(\xi^{S} a\right) N_{S}\left(\xi^{S}\right)}{D_{S}^{\prime}\left(\xi^{S}\right)} H_{1}^{(1)}\left(\xi^{S} r\right) e^{-i \omega t}-\pi i \frac{a^{2} \tau_{a}}{2 \mu} \sum_{\xi^{A}} \frac{J_{1}\left(\xi^{A} a\right) N_{A}\left(\xi^{A}\right)}{D_{A}^{\prime}\left(\xi^{A}\right)} H_{1}^{(1)}\left(\xi^{A} r\right) e^{-i \omega t}
$$

Analytical solution vs hybrid model (UX)

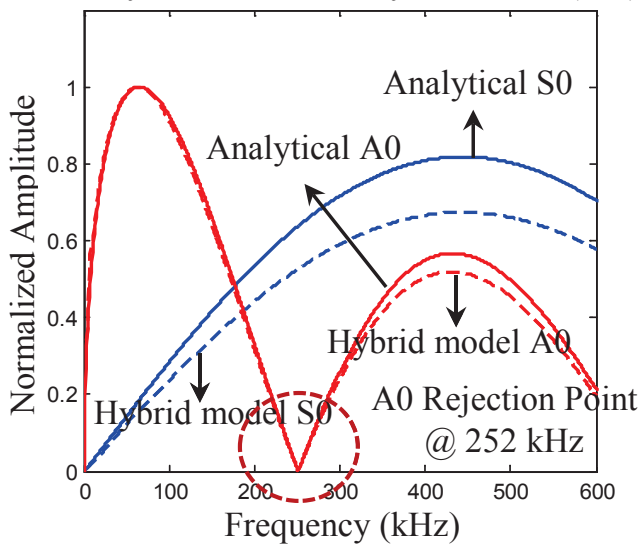

(a)

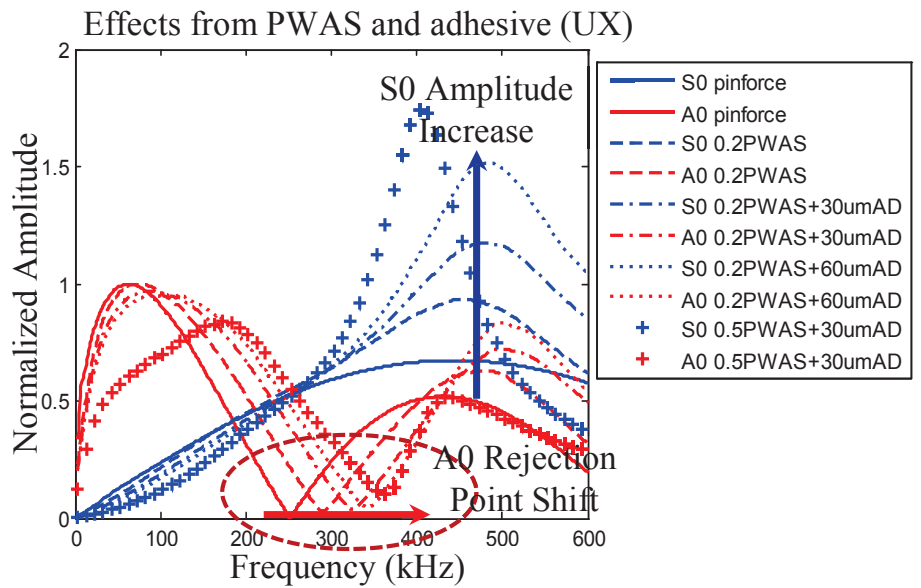

(b)

FIGURE 5. Tuning curves of case studies: (a) analytical solution vs hybrid model; (b) effects from PWAS and adhesive.

Figure 5a shows the comparison between the hybrid model with the analytical solution for the in-plane displacement. It should be noted only four elements (five FEM nodal solutions) were used across the thickness for this case study. It can be observed that the hybrid model compared well with the analytical solution especially for A0 mode. For high frequency range, the hybrid model deviates from the analytical solution, because more elements are needed to accurately depict the modeshape across the plate thickness. However, it can be noticed that the tuning behavior and the rejecting point (at $252 \mathrm{kHz}$ ) compared well. Figure $5 \mathrm{~b}$ shows different cases with various PWAS and adhesive layer thickness. It can be seen that the hybrid model is capable of capturing the local dynamics of PWAS and adhesive. When the PWAS and adhesive layer effects are considered, the A0 rejecting point shifts toward higher frequencies, and the rejecting effect becomes weaker. This rejecting point shift effect agrees with the observation in Ref [14]. At lower frequency range, the thicker PWAS/adhesive will reduce the excitability for both S0 and A0 modes, but the excitability of S0 becomes stronger at higher frequency range $(300 \mathrm{kHz}-600 \mathrm{kHz})$ due to the local dynamical effects. It should be noted that the dynamics of PWAS and adhesive cannot be fully captured by conventional pin force models. On the other hand, our hybrid model uses a small-size multi-physics FEM to handle this complex transducer structure interactions. 


\section{Circular PWAS on a 1-mm Thick Woven GFRP Plate}

Figure 6a shows the setup of the composite case study, which investigates the excitability of guided waves by a circular PWAS (7-mm diameter and $0.5 \mathrm{~mm}$ thick) bonded on a 1-mm thick woven GFRP plate. It also shows the 0 and 30 degree directions. Figure $6 \mathrm{~b}$ and Fig. $6 \mathrm{c}$ show the mode participation factor of S0, A0, and SH0 modes in the 0 and 30 degree directions respectively. It can be noticed that along 0 degree direction, only S0 and A0 wave modes are excited, while SH0 mode cannot be excited. In the 30 degree direction, not only S0 and A0 wave modes are excited, but also SH0 wave mode as well.

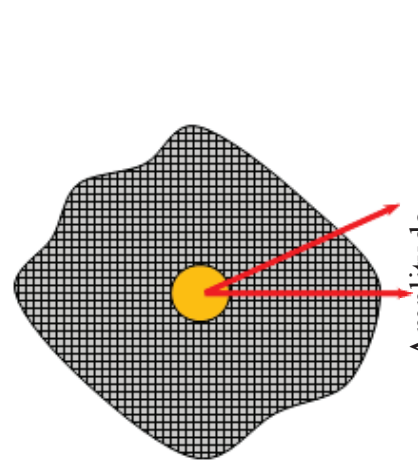

(a)

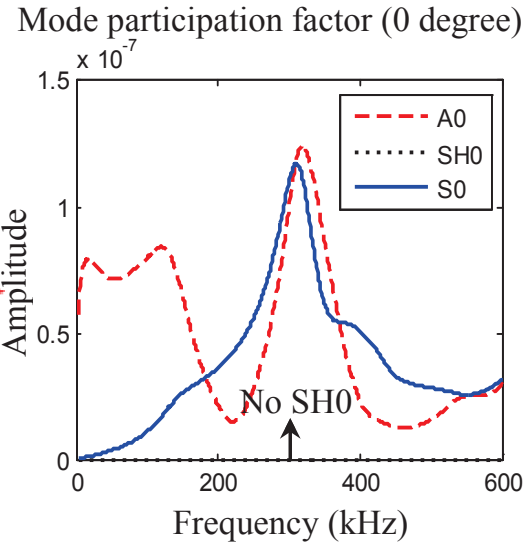

(b)

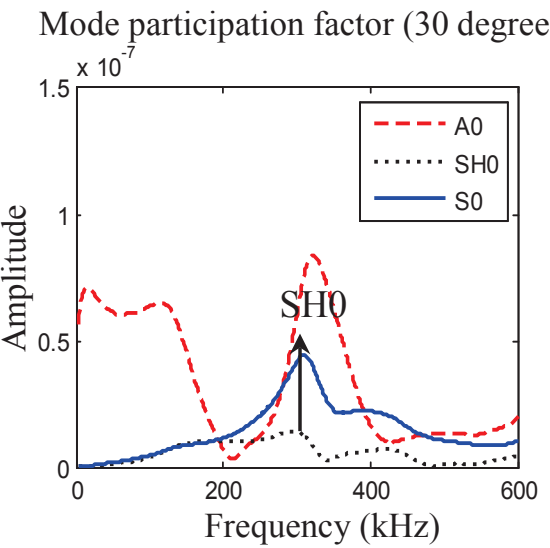

(c)

FIGURE 6. (a) Circular PWAS on the woven CFRP plate (arrows showing 0 and 30 degree directions); (b) mode participation factor for 0 degree direction; (c) mode participation factor for 30 degree direction.
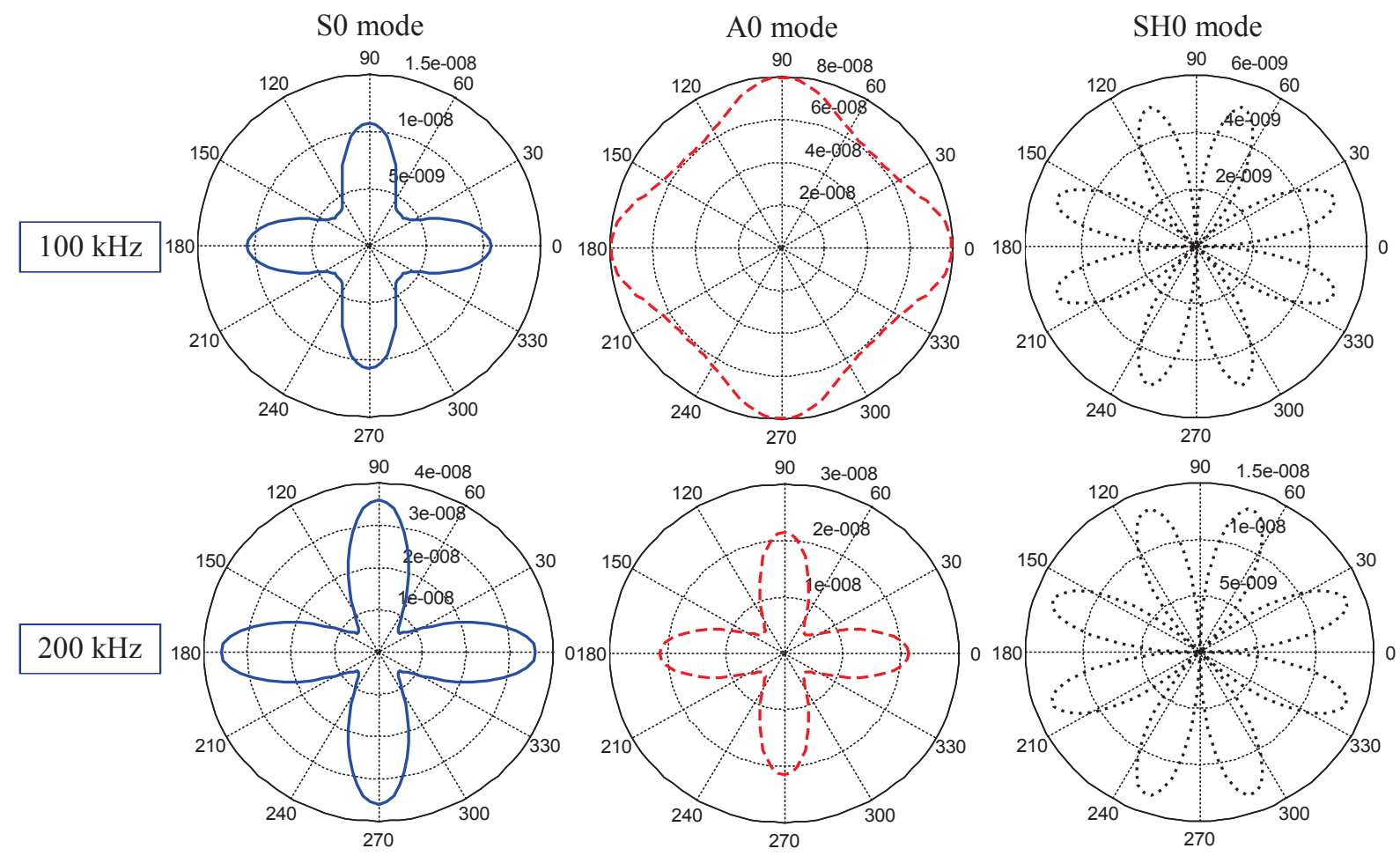

FIGURE 7. Directivity plots of mode participation factors for S0, A0, and SH0 modes excited by circular PWAS. 
Figure 7 shows directivity plots of mode participation factors for S0, A0, and SH0 modes excited by the circular PWAS at $100 \mathrm{kHz}$ and $200 \mathrm{kHz}$. It can be observed that strong S0 and A0 wave modes are excited along 0 and 90 directions, while no SH0 waves are excited in 0,45 , and 90 directions. The amplitude of S0 and A0 waves are weak in \pm 45 degree directions.

\section{Rectangular PWAS on a 1-mm Thick Woven GFRP Plate}

Figure 8a shows the setup of the composite case study, which investigates the excitability of guided waves by a rectangular PWAS ( $7 \mathrm{~mm}$ by $8 \mathrm{~mm}$ and $0.5 \mathrm{~mm}$ thick) bonded on a $1-\mathrm{mm}$ thick GFRP plate.

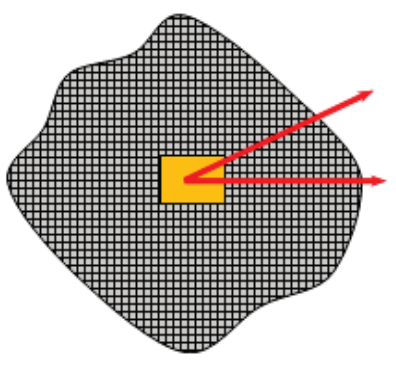

(a)

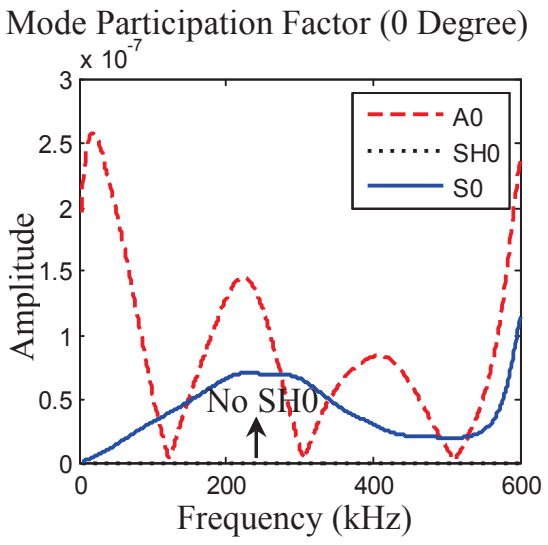

(b)

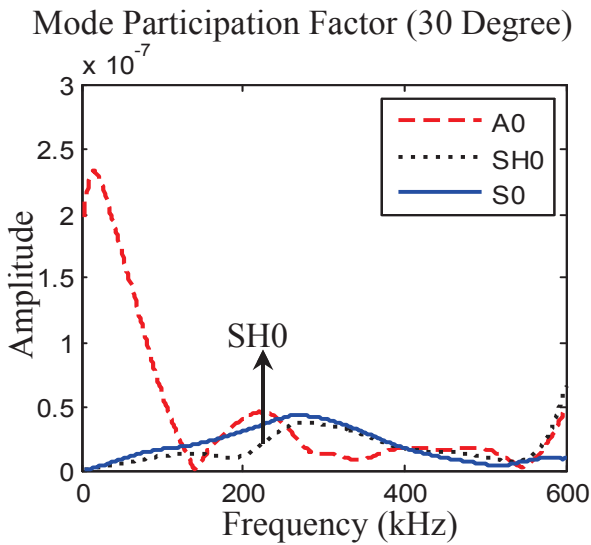

(c)

FIGURE 8. (a) Rectangular PWAS on the woven CFRP plate (arrows showing 0 and 30 degree directions); (b) mode participation factor for 0 degree direction; (c) mode participation factor for 30 degree direction. 

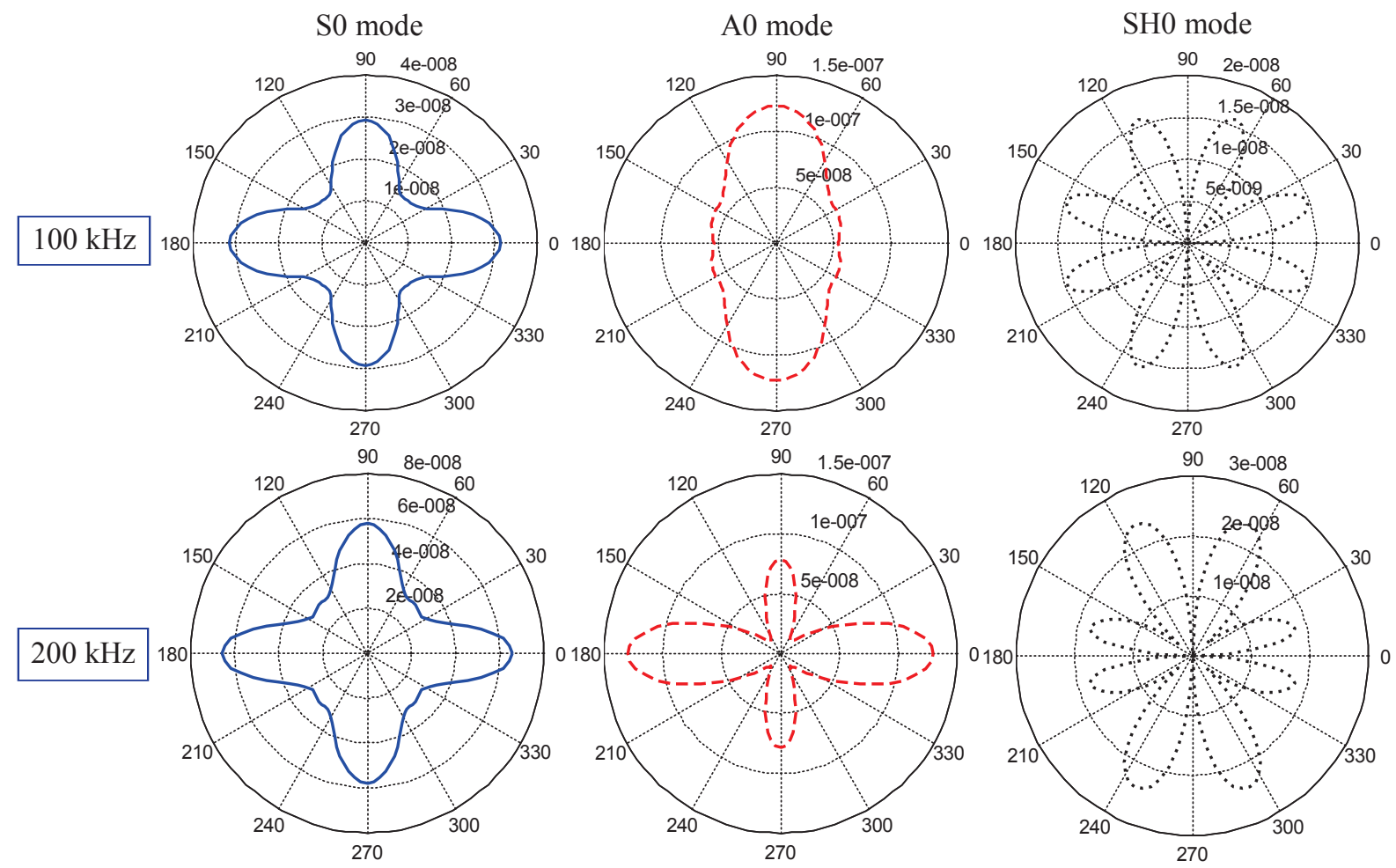

FIGURE 9. Directivity plots of mode participation factors for S0, A0, and SH0 modes excited by rectangular PWAS.

Figure $8 \mathrm{~b}$ and Fig. $8 \mathrm{c}$ show the mode participation factor of S0, A0, and SH0 modes in the 0 and 30 degree directions respectively for the rectangular PWAS case. It can be noticed that along 0 degree direction, only S0 and A0 wave modes are excited, while SH0 mode cannot be excited. In the 30 degree direction, not only S0 and A0 wave modes are excited, but also SH0 wave mode as well.

Figure 9 shows the directivity plots of $\mathrm{S} 0, \mathrm{~A} 0$, and $\mathrm{SH} 0$ wave modes excited by the rectangular PWAS at $100 \mathrm{kHz}$ and $200 \mathrm{kHz}$. Since the PWAS shape is rectangular with two different sizes in 0 and 90 directions, the tuning effects are different along these two directions. Such behavior is very obvious for the case of A0 wave mode. At $100 \mathrm{kHz}$, the strongest A0 waves are excited in 90 degree direction. However, at $200 \mathrm{kHz}$, the strongest $\mathrm{A} 0$ waves are excited in 0 degree direction. Strong S0 waves are excited along 0 and 90 degree directions, but the amplitudes in these two directions differ from each other. SH0 wave modes cannot be excited in 0 and 90 degree directions.

\section{CONCLUSIONS AND FUTURE WORK}

The small-size FEM with NRB can be used to extract guided wave excitation information in a highly efficient manner. The hybrid model comprehensive combines the FEM, SAFE method, and analytical solution. The hybrid model result compares well with analytical solution for the aluminum plate. This method can capture the dynamics of PWAS transducers and the adhesive layer. Only S0 and A0 waves can be excited by the extensional PWAS transducers in the aluminum plate. However, SH0 mode can be excited by the extensional PWAS in the woven GFRP composite plate. The case studies demonstrated the capability of this hybrid method to investigate the excitability of guided waves by complex dimension PWAS transducers on composite structures.

For future work, study of the influences on computational accuracy should be carried out. Experimental validation with pitch-catch and laser vibrometry should be performed. This work should be extended to combine with global analytical solution to form hybrid global local models for wave generation and propagation in composites. 


\section{ACKNOWLEDGMENTS}

Support from Office of Naval Research \# N00014-11-1-0271, Dr. Ignacio Perez, Technical Representative; Air Force Office of Scientific Research \#FA9550-11-1-0133, Dr. David Stargel, Program Manager; SPARC fellowship at University of South Carolina; are thankfully acknowledged.

\section{REFERENCES}

1. V. Giurgiutiu, Structural Health Monitoring with Piezoelectric Wafer Active Sensors, 2nd Edition, Elsevier Academic Press, 2014.

2. V. Giurgiutiu, "Tuned Lamb-Wave Excitation and Detection with Piezoelectric Wafer Active Sensors for Structural Health Monitoring," Journal of Intelligent Material Systems and Structures, vol. 16, no. 4, pp. 291306, 2005.

3. A. Raghavan and C. Cesnik, "Fintie-dimensional piezoelectric transducer modeling for guided wave based structural health monitoring," Smart Materials and Structures, vol. 14, pp. 1448-1461, 2005.

4. A. Raghavan and C. Cesnik, "3-D Elasticity-based Modeling of Anisotropic Piezocomposite Transducers for Guided Wave Structural Health Monitoring," in 47th AIAA/ASME/ASCE/AHS/ASC Structures, Structural Dynamics, and Materials Conference, Newport, 2006.

5. A. Velichko and P. Wilcox, "Modeling the excitatino of guided waves in generally anisotropic multi-layered media," The Journal of the Acoustical Society of America, vol. 121 (60), http://dx.doi.org/10.1121/1.2390674, 2007.

6. R. Schulte and C. Fritzen, "Simulation of wave propagation in damped composite structures with piezoelectric coupling," Journal of Theoretical and Applied Mechanics, vol. 49, pp. 879-903, 2011.

7. K. Nadella and C. Cesnik, "Effect of piezoelectric actuator modeling for wave generation in LISA," in SPIE conference proceeding, vol. 9064, doi:10.1117/12.2043514, 2014.

8. M. Gresil and V. Giurgiutiu, "Prediction of attenuated guided waves propagation in carbon fiber composites using Rayleigh damping model," Journal of Intelligent Material Systems and Structures, doi: 10.1177/1045389X14549870, 2014.

9. L. Gavric, "Computation of propagative waves in free rail using a fintie element technique," Journal of Sound and Vibration, vol. 85, pp. 531-543, 1995.

10. I. Bartoli, A. Marzani, F. Lanza de Scalea and E. Viala, "Modeling wave propagation in damped waveguides of arbitrary cross-section," Journal of Sound and Vibration, vol. 295, pp. 685-707, 2006.

11. Y. Shen, Structural health monitoring using linear and nonlinear ultrasonic guided waves, $\mathrm{PhD}$ dissertation University of South Carolina, 2014.

12. E. Glushkov, N. Glushkova, R. Lammering, A. Eremin and M. Neumann, "Lamb wave excitation and propagation in elastic plates with surface obstacles: proper choice of central frequencies," Smart Material and Structures, doi:10.1088/0964-1726/20/1/015020, 2011.

13. C. Ong, Y. Yang and Y. Wong, "The effects of adhesive on the electro-mechanical response of a piezoceramic transducer coupled smart system," in Proceeding of SPIE 5062:241-247, 2002.

14. G. Bottai and V. Giurgiutiu, "Exact shear-lag solution for guided waves tuning with piezoelectric wafter active sensors," AIAA Journal, vol. 50, pp. 2285-2294, 2012. 